Annuaire suisse de politique de développement

18 | 1999

La Suisse et l'action humanitaire

\title{
Évolution de l'aide humanitaire fournie par la confédération et les œuvres d'entraide suisses (de 1990 à 1997)
}

Gérard Perroulaz

\section{OpenEdition}

Journals

Édition électronique

URL : http://journals.openedition.org/aspd/665

DOI : 10.4000/aspd.665

ISSN : 1663-9669

Éditeur

Institut de hautes études internationales et du développement

Édition imprimée

Date de publication : 1 janvier 1999

Pagination : 9-23

ISSN : 1660-5934

\section{Référence électronique}

Gérard Perroulaz, « Évolution de l'aide humanitaire fournie par la confédération et les œuvres d'entraide suisses (de 1990 à 1997) », Annuaire suisse de politique de développement [En ligne], 18 |

1999, mis en ligne le 14 juillet 2012, consulté le 08 septembre 2020. URL : http://

journals.openedition.org/aspd/665; DOI : https://doi.org/10.4000/aspd.665 


\title{
ÉVOLUTION DE L'AIDE HUMANITAIRE FOURNIE PAR LA CONFÉDÉRATION ET LES EUUVRES D'ENTRAIDE SUISSES (DE 1990 À 1997)
}

\author{
Gérard Perroulaz*
}

\section{1. ÉVOLUTION DE L'AIDE HUMANITAIRE DES PAYS DU CAD'}

L'OCDE publie des estimations globales de l'aide humanitaire (voir encadré). L'aide d'urgence avait fortement progressé à partir de 1990, pour atteindre un point culminant en 1994. Cette année-là, le CAD (Comité d'aide au développement de l'OCDE) évaluait le montant total de l'aide d'urgence (y compris le secours aux réfugiés) fournie par les pays membres à plus de 3,5 milliards de dollars. L'aide d'urgence multilatérale passant par l'UNICEF, le HCR, le PAM et l'Union européenne s'élevait pour sa part à près de 3 milliards de dollars. Les dépenses militaires (non comprises dans l'APD) de maintien de la paix ont elles aussi beaucoup augmenté ces dernières années. Ces opérations de maintien de la paix se sont élevées à près de 3,5 milliards de dollars en 1994, dont près de la moitié pour la seule région de l'ex-Yougoslavie ${ }^{2}$. En 1996, l'aide d'urgence se chiffrait à 3 milliards de dollars sur le plan bilatéral et 2,5 milliards de dollars sur le plan multilatéral, sans compter les 3 milliards de dollars que coûtent les opérations de maintien de la paix (non comptabilisés dans l'APD). L'aide humanitaire totale serait donc passée de 10 milliards à 8,5 milliards de dollars de 1994 à 1996.

\section{2. ÉVOLUTION DE L'AIDE HUMANITAIRE DE LA CONFÉDÉRATION}

Le mandat de la Confédération est décrit dans la loi fédérale de 1976 sur la coopération au développement et l'aide humanitaire internationales: «L'aide humanitaire a pour but de contribuer, par des mesures de prévention ou de secours, à la sauvegarde de la vie humaine lorsqu'elle est menacée ainsi qu'au soulagement des souffrances; elle est notamment destinée aux populations victimes d'une catastrophe naturelle ou d'un conflit armé.» Le texte de la partie «Revue» de cet Annuaire donne des informations sur l'organisation et les moyens d'intervention dans le cadre de cet instrument de la coopération internationale, avec les données récentes sur l'aide humanitaire de la Confédération.

Le schéma suivant résume les formes d'intervention de l'aide humanitaire. La Confédération accorde son aide par deux voies: les interventions directes, notamment celles du Corps suisse d'aide en cas de catastrophe (ASC, 15\% environ des versements), et le soutien apporté aux organisations partenaires

* Chargé de recherche, IUED.

1. $\mathrm{CAD}=$ Comité d'aide au développement, composé de 21 pays.

2. Source : OCDE, Coopération pour le développement. Efforts et politiques des membres du Comité d'aide au développement, rapport 1996, OCDE, Paris, 1997, pp. 110-112. 
(organisations multilatérales, CICR et œuvres d'entraide suisses). La Confédération dispose de quatre moyens d'intervention: personnel de l'ASC, contributions financières, livraisons de denrées alimentaires et de matériel. Les domaines d'activités sont:

口 la prévention: observation de l'activité volcanique en Amérique centrale par exemple;

\ le sauvetage: dégagement et assistance aux blessés, sauvetage de personnes ensevelies après un tremblement de terre;

- la survie: approvisionnement en eau potable, distribution de nourriture, montage de tentes et d'abris, assistance médicale;

- la reconstruction: remise en état des infrastructures (routes, ponts, etc.), reconstruction de bâtiments publics (hôpitaux, foyers d'accueil, écoles) et des habitations, programmes de santé.

\section{Schéma n¹: Formes d'intervention de l'aide humanitaire}

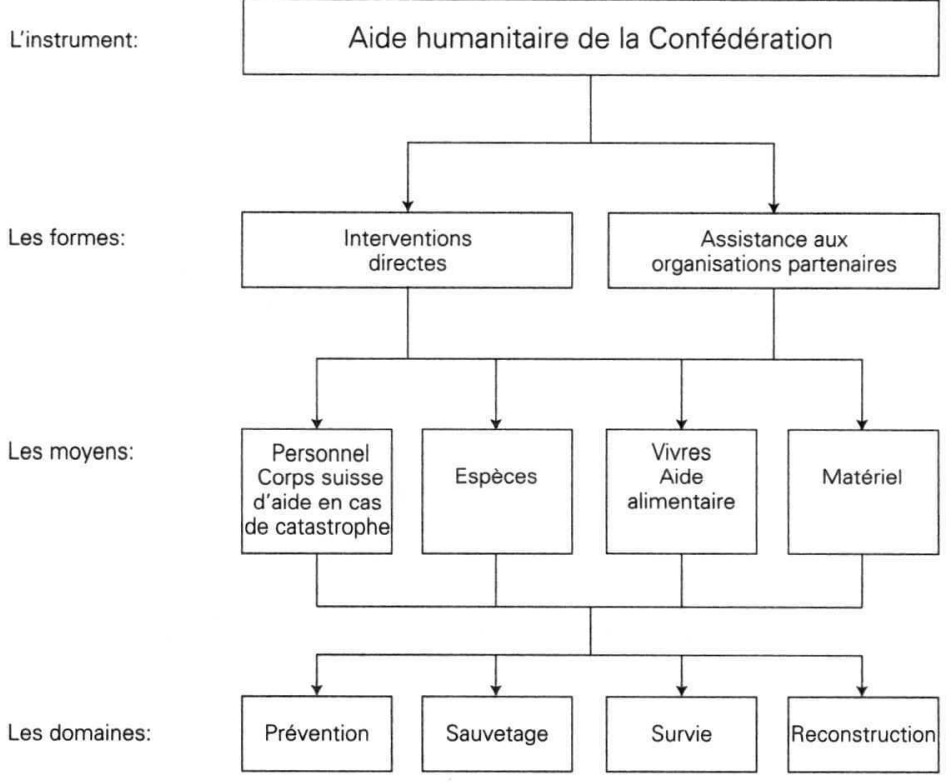

Source: "Message du Conseil fédéral sur la continuation de l'aide humanitaire internationale de la Confédération " du 20 novembre 1996, Berne, message 96.092.

Le public a souvent l'impression que l'aide humanitaire a explosé ces dernières années, cela au détriment de la coopération au développement à plus long terme. L'analyse de l'évolution récente de l'aide humanitaire fournie par la Confédération contredit cette impression, du moins en ce qui concerne l'aide comprise dans l'APD (aide publique au développement). La part de l'aide humanitaire dans l'APD a oscillé depuis 1986 entre $17,8 \%$ et $22 \%$ de l'APD totale, et est même en diminution depuis 1991 (en passant de 20,4\% de l'APD en 1991 à $17,8 \%$ en 1997). 


\section{Difficultés méthodologiques pour l'analyse de l'aide humanitaire}

L'analyse de l'évolution récente de l'aide humanitaire se heurte à certaines difficultés d'interprétation des données statistiques: il n'y a aucun agrégat statistique exhaustif sur l'aide humanitaire et la limite entre coopération au développement à plus long terme et aide d'urgence devient de plus en plus floue.

Plusieurs domaines de l'aide au développement se situent à la frontière entre aide humanitaire et coopération au développement: prévention des conflits, aide aux enfants de la rue, distribution de lait et de nourriture dans le cadre d'appui à des écoles, opérations de maintien de la paix des Nations Unies. A la fin d'un conflit, l'appui aux efforts de reconstruction revêt une importance de plus en plus grande. Ces efforts ne se limitent pas à la remise en état d'infrastructures de base, à la reconstruction d'immeubles, d'hôpitaux et d'écoles, mais couvrent un champ de plus en plus vaste: opérations de déminage, retour des personnes déplacées et des réfugiés, réinsertion des soldats démobilisés ou de rebelles dans la vie économique, appui au processus de démocratisation (supervision des élections, renforcement des systèmes judiciaires, promotion et protection des droits de l'homme, reconversion des installations de production de matériel militaire vers des activités civiles).

Les statistiques du Comité d'aide au développement de I'OCDE ne permettent pas de connaître le montant précis de l'aide humanitaire. L'OCDE distingue l'aide apportée aux pays en développement, comprise dans les statistiques d'aide publique au développement, de celle fournie aux pays en transition, incluse dans l'agrégat d'aide publique (pays de l'Europe centrale et orientale, pays en développement économiquement plus avancés). II est très difficile de comparer l'aide humanitaire fournie par les différents pays du CAD puisque les statistiques publiées par le CAD ne permettent pas d'isoler tous les éléments de cette aide dans un agrégat séparé d'aide humanitaire.

Dans les statistiques du CAD, on peut isoler les éléments suivants de l'aide humanitaire:

- Aide alimentaire: livraisons de denrées destinées à I'alimentation humaine effectuées dans le cadre de secours d'urgence, contributions en espèces pour l'achat de denrées alimentaires, frais de transport des produits, livraisons de produits intermédiaires dans le cadre des programmes d'aide alimentaire (produits d'alimentation animale et moyens de production agricoles tels qu'engrais, semences). L'aide multilatérale dans ce domaine passe le plus souvent par le Programme alimentaire mondial (PAM). Dans les statistiques du CAD, I'aide alimentaire comprend aussi I'aide alimentaire qui serait fournie dans le cadre de programmes de coopération au développement.

- Secours d'urgence lors d'une catastrophe naturelle ou provoquée par des actions humaines.

- Les dépenses pour l'hébergement temporaire des réfugiés dans les pays du Nord peuvent (si les pays le souhaitent) être comptabilisées dans I'APD pendant la première année de l'accueil. La plupart des pays, dont la Suisse, ne comptabilisent pas ces dépenses dans leur APD. Toutefois, un nombre croissant de pays font progressivement usage de cette possibilité. Les dépenses effectuées en vue de la réinstallation de réfugiés dans un pays bénéficiaire de l'aide peuvent être incluses dans I'APD.

- Dépenses du secteur public pour le soutien des réfugiés ou des personnes déplacées dans les pays en développement.

Pour une analyse complète de l'aide humanitaire, il faudrait aussi pouvoir, ce qui n'est malheureusement pas le cas, isoler les éléments suivants:

- Aide multilatérale (contributions aux organisations multilatérales actives dans l'action humanitaire). II n'est pas possible d'isoler dans les statistiques du CAD les montants d'aide multilatérale destinés aux organisations internationales actives uniquement ou surtout dans les actions humanitaires des montants destinés pour la coopération à plus long terme. Les contributions au HCR, à I'UNRWA* sont par exemple englobées dans la somme des contributions au Système des Nations Unies (avec tous les autres organismes et fonds opérationnels). Certaines organisations multilatérales travaillent en outre dans les deux domaines, sans qu'il soit possible d'isoler les deux aspects (contributions à l'OMS ou à I'UNICEF par exemple). 
- Aide aux organisations privées internationales. Les contributions de la Suisse pour le CICR sont comptabilisées dans l'aide bilatérale. Les contributions aux organisations internationales privées comprennent les contributions au CICR, mais aussi une série d'organisations privées actives dans des projets à plus long terme (Entraide universitaire mondiale ou Institut panafricain pour le développement par exemple).

Certains aspects liés à l'aide humanitaire ne sont pas comptabilisés dans I'APD:

- les dépenses des pays pour l'accueil des réfugiés dans les pays du Nord ne sont pas notifiées en tant qu'APD après la première année d'accueil dans ce pays;

- les dépenses des pays pour les forces de maintien de la paix ne sont pas incluses dans l'APD (mais dans les budgets militaires).

* UNRWA: Office de secours et de travaux des Nations Unies pour les réfugiés de Palestine dans le Proche-Orient.

\section{PRINCIPALES RÉGIONS BÉNÉFICIAIRES}

\section{DE L'AIDE HUMANITAIRE DE LA CONFÉDÉRATION DE 1990 À 1997}

Les montants d'aide humanitaire sont plus volatils d'une année à l'autre que la coopération au développement, cela au gré des catastrophes et des nouveaux conflits qui apparaissent dans l'une ou l'autre des régions du monde. C'est pourquoi l'analyse de l'évolution de l'aide humanitaire d'une année à l'autre n'a pas beaucoup de sens, les variations pouvant être très fortes pour un pays donné. Pour mieux analyser les caractéristiques de l'aide suisse, nous avons ainsi regroupé l'aide fournie de 1990 à 1997. Le tableau de l'annexe I relève les montants d'aide humanitaire de la Confédération pour des actions humanitaires durant ces années. Il souligne les principaux pays ou régions bénéficiaires de l'aide humanitaire (pays en développement et pays de l'Europe centrale et orientale).

Plusieurs aspects peuvent ressortir des données chiffrées de l'annexe. Parmi les remarques générales, on peut relever que dans l'ensemble l'aide humanitaire a été utilisée plutôt pour soulager les drames provoqués par l'action humaine que pour aider les victimes de catastrophes naturelles. Ces dernières années, les conflits interethniques et les guerres civiles ont entraîné plus de drames humains que les catastrophes naturelles (inondations, passages de cyclones, sécheresses, tremblements de terre, éruptions volcaniques, glissements de terrain). Si l'aide fournie dépend bien sûr de l'actualité des catastrophes et conflits divers, on trouve néanmoins, parmi les principaux pays bénéficiaires de l'aide humanitaire suisse, quelques pays de concentration de la coopération au développement à plus long terme (Madagascar, Mozambique, Pérou, Tanzanie).

Le graphique suivant montre la répartition de l'aide humanitaire par régions. $43 \%$ de l'aide bilatérale ne peuvent pas être ventilés géographiquement; ce montant recouvre surtout les contributions de la Suisse aux opérations du CICR. Le continent africain est le premier continent bénéficiaire de l'aide humanitaire de la Suisse. 


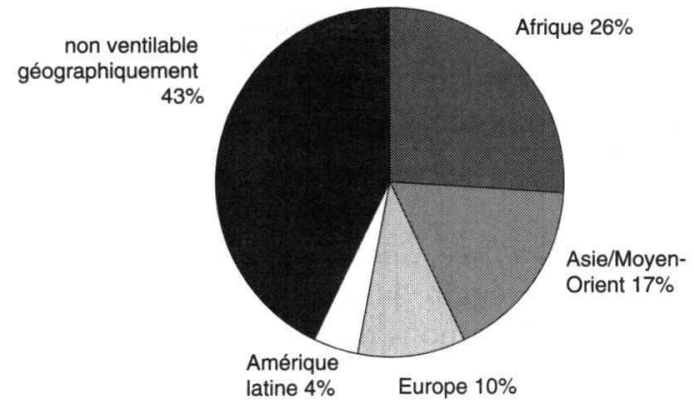

Graphique $n^{\circ} 2$ : Les dix premiers pays/régions bénéficiaires de l'aide humanitaire de la Confédération (versements totaux de 1990 à 1997, en millions de francs)

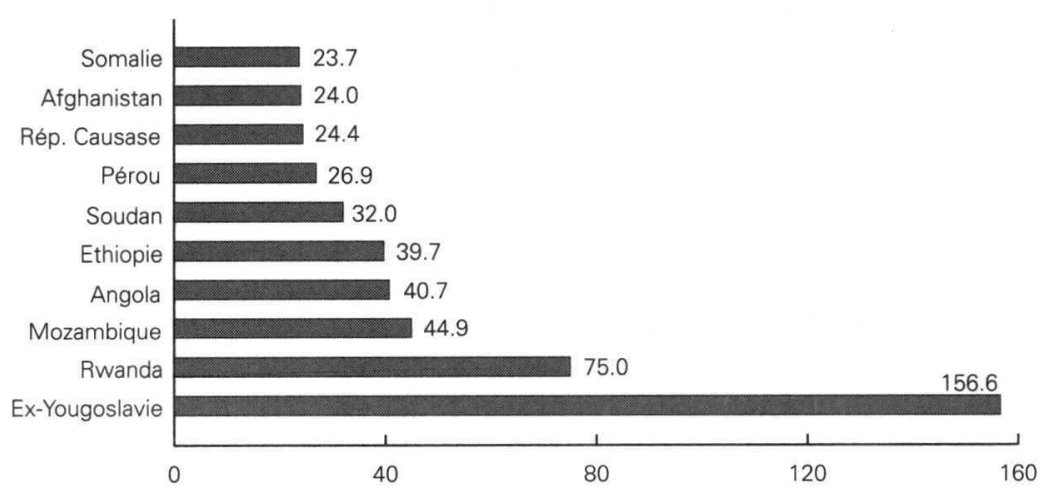

Sources des graphiques 1 et 2: DDC, Coopération internationale de la Suisse - Aide humanitaire, DDC, Berne, rapports annuels 1996 et 1997; IUED, Aide suisse aux pays en développement et aux pays de l'Europe orientale, IUED, Genève, éditions de 1991 à 1997.

Dans une analyse par régions plus fine, on peut relever les principales régions touchées par des catastrophes humanitaires où la Suisse a apporté une grande partie de son aide. L'analyse ci-dessous porte sur la période de 1990 à 1997.

\section{$\square$ En Europe}

\section{Etats de l'ex-Yougoslavie}

Jamais la Suisse n'avait versé autant de ressources dans une région en crise que dans les Etats en conflit issus de l'ex-Yougoslavie. L'APD humanitaire fournie par la Confédération a atteint 157 millions de francs de 1991 à 1997 (aide versée surtout entre 1992 à 1995). A cela s'ajoutent les aspects non compris dans l'APD: aide aux réfugiés en Suisse, une partie de l'aide au retour des réfugiés bosniaques versée par l'Office fédéral des réfugiés, actions de la Suisse dans le cadre de l'OSCE (Organisation pour la sécurité et la coopération en Europe), programmes d'aide à la reconstruction compris dans la coopération au développement, soutien au processus électoral en Bosnie, missions des bérets jaunes, soutien à des médias indépendants. Au total, les dépenses de la Suisse en faveur 
de ces Etats se seraient élevées à 1,2 milliard de dollars de 1991 à 1996. Depuis 1996, l'aide humanitaire baisse au profit d'activités de reconstruction à plus long terme (comprises dans la coopération au développement).

Russie

L'aide humanitaire destinée à la Russie s'est élevée à 14 millions de francs sur la période étudiée (dont 9,4 millions en 1995 et 1996).

\section{$\square$ En Afrique}

Corne de l'Afrique (Ethiopie, Erythrée, Somalie et Soudan)

C'est avec l'Afrique australe la région du continent qui a bénéficié le plus de l'aide fournie par la Suisse. 51,5 millions de francs ont été versés pour les victimes du conflit en Ethiopie et la nouvelle Erythrée (surtout de 1994 à 1996); 55,7 millions ont été acheminés pour les victimes des drames de la Somalie et du Soudan (surtout entre 1990 et 1993).

\section{Rwanda/région des Grands Lacs}

La plupart des programmes de coopération dans ce pays ont été bouleversés suite aux massacres. L'aide humanitaire a alors pris une importance très grande. Près de 82 millions de francs ont été versés par la Suisse au Burundi et au Rwanda (surtout depuis 1993).

\section{Mozambique/Malawi}

56 millions de francs d'aide humanitaire ont été versés à cette région, surtout pour venir en aide aux réfugiés mozambicains. L'aide humanitaire au Mozambique s'inscrit dans le cadre de la coopération au développement avec ce pays de concentration de l'aide suisse.

\section{Angola}

41 millions de francs d'aide humanitaire ont été versés à la population angolaise.

\section{Afrique du Nord}

L'aide humanitaire s'est surtout concentrée sur l'Algérie, avec des montants relativement modestes (10 millions de francs, dont près de la moitié en 1991). La Confédération fournit surtout de l'aide alimentaire par le biais des œuvres d'entraide (dont une partie pour des camps de réfugiés sahraouis).

Toute une série de pays africains ont bénéficié de l'aide suisse pour des montants situés la plupart du temps entre 10 et 21 millions de francs pour chacun d'eux (par ordre décroissant des montants): Madagascar, avec 21 millions de francs d'aide versés de 1990 à 1997 (pays de concentration de l'aide suisse), République démocratique du Congo (16 millions de francs), Tanzanie, Liberia, Kenya, Afrique du Sud.

\section{$\square$ Au Proche et Moyen-Orient}

Dans cette région, l'aide humanitaire s'est concentrée sur les territoires israéliens et palestiniens, le Liban et la Jordanie.

\section{$\square$ En Asie}

On peut relever les principales régions suivantes pour l'intervention humanitaire de la Confédération: républiques du Caucase (Arménie, Azerbaïdjan, Géorgie, 
avec au total des versements de plus de 24 millions de francs, surtout depuis 1994), victimes des guerres en Afghanistan (24 millions d'aide), conflits et tremblements de terre en Iran/Irak (21 millions de francs versés de 1990 à 1997), région de 1'Inde et du Bangladesh (plus de 19 millions de francs versés dans cette région), Sri Lanka en guerre civile (17 millions de francs), Cambodge (14 millions de francs). Depuis 1995, la Suisse verse une aide alimentaire substantielle à la Corée du Nord (13 millions de francs de 1995 à 1997).

\section{$\square$ En Amérique latine}

L'aide destinée à cette région a été relativement faible et concerne étonnamment surtout des pays avec lesquels la Confédération et les ONG entretiennent aussi des programmes de coopération au développement à long terme (importance de l'aide au Pérou, Brésil et Chili).

\section{Aide à l'Amérique centrale}

Région de concentration de l'aide suisse, l'Amérique centrale a reçu l'aide humanitaire surtout suite à des conflits (guerre civile du Salvador, conflits au Nicaragua), notamment pour l'aide aux personnes déplacées et réfugiées dans la région. Cette aide concerne pourtant aussi des catastrophes naturelles (tremblement de terre au Nicaragua en 1992 par exemple).

Haïti

Près de 10 millions de francs ont été versés dans ce pays.

\section{AIDE HUMANITAIRE GÉRÉE PAR LES EEUVRES D'ENTRAIDE}

Les dons des ONG en faveur de l'aide humanitaire (appelés dons privés) sont pris en compte dans les statistiques du CAD lorsqu'ils sont versés par une ONG du Nord à une ONG du Sud ou aux populations bénéficiaires (aide monétaire ou en nature). Sous «dons privés» ne sont en revanche pas comptabilisés tous les autres paiements de source privée entrant dans la définition des «transferts privés » au sens de la balance des paiements (par exemple les envois de fonds des travailleurs immigrés et des réfugiés en Europe à leurs familles).

Beaucoup d'ONG sont actives dans l'aide humanitaire, mais les statistiques de l'aide humanitaire des ONG sont à analyser avec prudence, car ce sont les ONG, en répondant à des enquêtes annuelles, qui déterminent ce qu'elles considèrent relever plutôt de l'aide d'urgence ou de la coopération au développement. Si l'on considère que la frontière entre ces domaines est assez floue, la notion d'aide humanitaire peut varier d'un responsable d'ONG à un autre.

\section{$\square$ Montant total de l'aide humanitaire gérée par des $O N G$}

Le financement des actions humanitaires des ONG provient des collectes des ONG auprès du public, de grandes collectes nationales d'organisations telles que la Chaîne du bonheur, des cantons et communes. En 1996 les dépenses totales des ONG pour des actions humanitaires dans le Sud et à l'Est se sont ainsi élevées à plus de 95,2 millions de francs, dont 62 millions ont été financés grâce aux collectes réalisées auprès de la population et 30 millions grâce aux contributions de la Confédération. A ces sommes s'ajoutent 3 millions de francs d'aide humanitaire des cantons et communes suisses, acheminés par le biais des 
ONG. Les données statistiques concernant le financement des actions d'ONG suisses provenant de l'étranger sont très lacunaires (financement d'organisations sœurs ou mères à l'étranger, financement de l'Union européenne ou d'Etats étrangers).

\section{$\square$ Aide humanitaire de la Confédération acheminée par le biais des ONG}

Une partie de l'aide humanitaire de la Confédération (APD) est acheminée par le canal des ONG, c'est-à-dire est administrée par les ONG avec le financement ou la fourniture de vivres par la Confédération (lait en poudre par exemple). Ainsi 14 à $16 \%$ de l'aide humanitaire de la Confédération passent par le biais des ONG. En 1997, l'aide humanitaire de la Confédération transitant par les ONG s'élevait à 32,3 millions. L'aide a été acheminée surtout par le biais des ONG suivantes: Caritas Suisse (7,9 millions de francs en 1997), Croix-Rouge suisse (6,8 millions de francs), Terre des hommes Lausanne (4 millions de francs), Armée du Salut, Entraide protestante suisse, Médecins sans frontières et Terre des hommes Suisse - Genève (voir deuxième colonne du tableau).

Tableau $n^{\circ} 1$ : Principales organisations privées suisses actives dans l'aide humanitaire

\begin{tabular}{|c|c|c|}
\hline \multirow{2}{*}{$\begin{array}{l}\text { Principales ONG } \\
\text { actives dans I'aide } \\
\text { humanitaire }\end{array}$} & \multicolumn{2}{|c|}{ En milliers de francs } \\
\hline & $\begin{array}{c}\text { Fonds privés } \\
\text { en } 1997\end{array}$ & $\begin{array}{c}\text { Contributions } \\
\text { Confédération en } 1997\end{array}$ \\
\hline Caritas Suisse & 15046 & 7904 \\
\hline Terre des hommes Lausanne & 9116 & 4010 \\
\hline Croix-Rouge suisse & 7888 & 6804 \\
\hline EPER Entraide protestante & 3223 & 1503 \\
\hline Seraphisches Liebeswerk & 2543 & \\
\hline Mediswiss & 1933 & \\
\hline Kroatisches humanitäres Forum & 1679 & 20 \\
\hline Basel Hilft & 1603 & \\
\hline Christian Solidarity Internat. & 1381 & \\
\hline St. Petrus-Claver-Solidarität & 1108 & \\
\hline Fondation Novartis & 860 & \\
\hline Heilige Familie Werthenstein & 810 & \\
\hline Amis de Sœur Emmanuelle & 744 & \\
\hline ADRA, Adventistes & 678 & 484 \\
\hline Médecins sans frontières & 518 & 1200 \\
\hline OSEO/SAH & 517 & 202 \\
\hline Mariannhiller Missionare & 509 & \\
\hline Medair & 312 & 1100 \\
\hline Armée du Salut & 130 & 1607 \\
\hline Terre des hommes Suisse - Genève & 81 & 1114 \\
\hline Basler Mission & 43 & 397 \\
\hline Comité de soutien au peuple sahraoui & 36 & 574 \\
\hline Solidarité Tiers Monde & 0 & 638 \\
\hline Enfants du monde & 0 & 625 \\
\hline Fondation Agha Khan & 0 & 800 \\
\hline ONG ci-dessus & 51070 & 28982 \\
\hline Autres organisations & 6832 & 3278 \\
\hline Total aide des ONG & 57902 & 32260 \\
\hline
\end{tabular}

Source: IUED, Aide suisse aux pays en développement et aux pays de l'Europe orientale 1997/98, IUED, Genève, 1999. 
Grâce aux collectes auprès du public, les ONG ont pu financer des actions humanitaires pour un montant de 52 millions de francs en 1997 dans les pays en développement et pour 6 millions de francs dans les pays de l'Europe centrale et orientale. Le tableau ci-dessus donne la liste des principales ONG actives dans l'aide humanitaire, avec, en première colonne, les actions financées grâce aux collectes auprès du public et, dans la deuxième colonne, les montants financés grâce aux contributions de la Confédération.

\section{$\square$ Le rôle de la Chaîne du bonheur}

N'apparaissent pas dans le tableau certaines organisations qui jouent un rôle très important dans la récolte de fonds auprès du public mais qui ne gèrent pas ellesmêmes des actions humanitaires. Les collectes de la Chaîne du bonheur par exemple ont permis de réunir près de 10,2 millions de francs en 1996. Ces fonds sont reversés à des œuvres d'entraide pour financer les actions de ces organisations. En 1997, la Chaîne du bonheur a ainsi versé 8,5 millions de francs aux œuvres d'entraide pour des actions dans les pays en développement et 1,25 million de francs pour des actions dans les pays de l'Europe centrale et orientale. En 1997, les œuvres d'entraide qui ont obtenu les contributions les plus importantes par le biais de la Chaîne du bonheur ont été les suivantes: Caritas Suisse (4,8 millions de francs), Croix-Rouge suisse (3,5), Entraide protestante $(2,1)$ et Terre des hommes Lausanne $(1,1)$. Il est intéressant de relever qu'une partie de l'argent récolté par la Chaîne du bonheur suite à des catastrophes n'est pas affectée à l'aide d'urgence classique, mais permet une reconstruction et une aide à plus long terme dans les régions touchées. L'argent récolté lors de collectes peut ainsi être distribué pour des actions échelonnées sur plusieurs années.

\section{$\square$ Principaux pays bénéficiaires de l'aide des $O N G$}

Les graphiques suivants montrent les principales régions bénéficiaires de l'action humanitaire des ONG suisses de 1990 à 1997. L'annexe II donne le détail de l'aide des ONG pour les principaux pays bénéficiaires année après année. La part de l'aide humanitaire versée aux pays de l'Europe de l'Est et aux pays asiatiques est plus importante que dans le cas de l'aide de la Confédération. Certains des pays principaux bénéficiaires de l'aide des ONG se trouvent aussi dans la liste des principaux bénéficiaires de l'aide de la Confédération (ex-Yougoslavie, Rwanda, Somalie et Soudan, Arménie). L'aide des ONG aux Etats de l'exYougoslavie, d'un montant total de 89 millions de francs, s'ajoute à l'aide de la Confédération de 157 millions de francs, sans compter les versements privés de l'importante communauté bosniaque résidant en Suisse (dons à leurs familles et proches et à certaines organisations qui ne répondent pas aux questionnaires sur les versements à l'étranger). L'aide à l'Irak concerne surtout l'aide humanitaire aux Kurdes (grâce notamment aux importants dons récoltés par la Chaîne du bonheur). 
Graphique $n^{\circ} 3$ : Répartition de l'aide humanitaire des ONG par continent (1997, en \% du total)

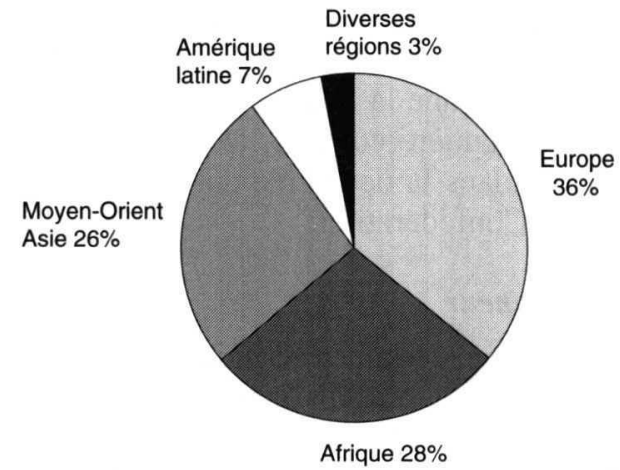

\section{Graphique $n^{\circ} 4$ : Les dix premiers pays/régions bénéficiaires de l'aide humanitaire des ONG (actions financées par les fonds propres) (versements totaux de 1990 à 1997, en millions de francs)}

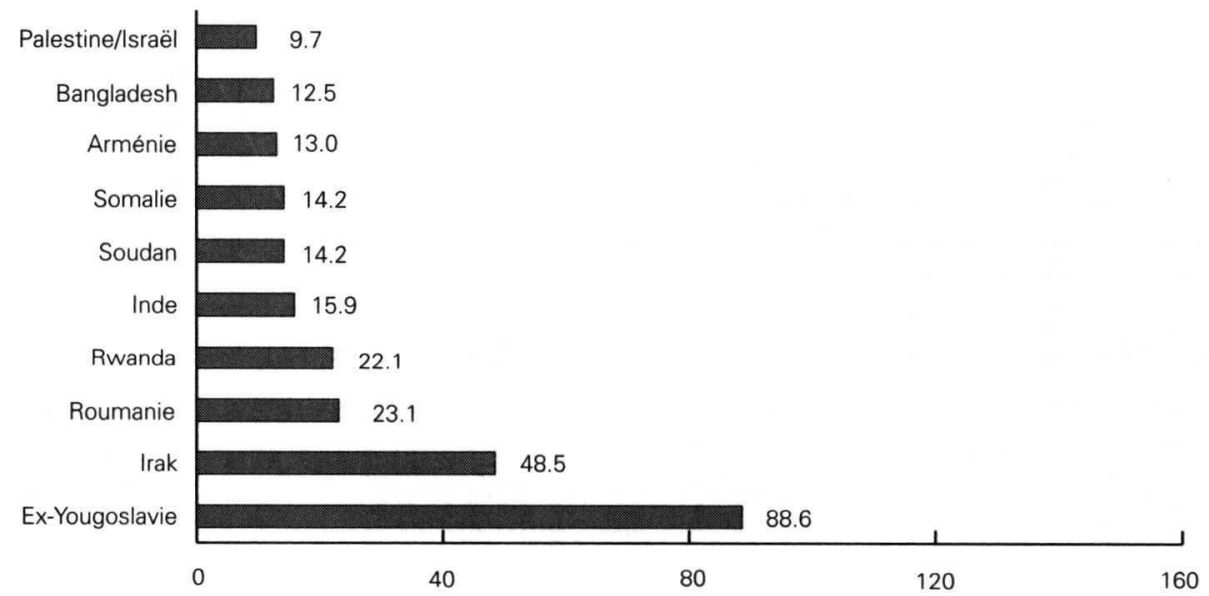

Source des graphiques 3 et 4 : calculé à partir des données statistiques parues dans la publication de l'IUED Aide suisse aux pays en développement et aux pays de l'Europe orientale, IUED, Genève, éditions 1991 à $1997 / 98$.

\section{$\square$ Rôle des collectes auprès du public}

Le rôle des ONG est très important pour mobiliser l'opinion publique et récolter des fonds. Une grande partie de l'argent récolté auprès du public lors de collectes diverses est liée d'une manière ou d'une autre à l'écho médiatique de certaines catastrophes. Les rapports entre ONG et médias sont ambigus, voire incestueux. Les ONG ont besoin d'un écho médiatique sur certaines catastrophes pour pouvoir ensuite lancer des campagnes de récoltes de fonds. On peut parfois se demander si ce ne sont pas les messages les plus catastrophistes qui suscitent les plus grandes recettes pour les ONG. Les médias prêtent généralement une attention plus grande aux situations d'urgence qu'aux situations de développement, car le côté spectaculaire provoque une émotion et une attention 
plus soutenues du téléspectateur. En 1997, un article du magazine Mosquito portait ce titre provocateur: «Donnez-nous notre catastrophe quotidienne» ${ }^{3}$. La répétition de catastrophes humanitaires provoquées par l'homme et les conflits peuvent pourtant à long terme entraîner une certaine lassitude du donateur.

Certains thèmes sont plus porteurs que d'autres lorsque l'on veut récolter de l'argent auprès du public. Récolter de l'argent pour aider les affamés ou les enfants de la rue est incontestablement plus facile que de récolter de l'argent pour aider des réfugiés (ici ou ailleurs) ou soutenir l'action de syndicats du Tiers Monde. Il suffit de voir l'utilisation massive des photos d'enfants en détresse dans les envois publicitaires et les journaux d'organisations humanitaires pour comprendre que de telles illustrations sont sûrement plus porteuses pour récolter des dons que l'image d'un homme de couleur. Autre facteur de motivation pour le donateur, près de la moitié de l'argent récolté par les œuvres d'entraide en Suisse l'est par des organismes missionnaires ou proches des Eglises. Si les missions ne travaillent bien sûr pas uniquement dans l'aide d'urgence, mais soutiennent aussi des projets de développement à plus long terme, ce fait nous révèle toutefois une partie des motivations des donateurs (importance du don par charité chrétienne).

\section{$\square$ Profusion d'acteurs de l'humanitaire travaillant sur le même terrain}

Des centaines d'ONG suisses (ou branches suisses d'organisations internationales) sont actives sur le marché humanitaire, ce qui doit dérouter plus d'un donateur confronté à la profusion d'envois publicitaires dans la boîte aux lettres. On imagine aussi les problèmes de coordination sur le terrain. Dans une région comme la Bosnie, le pays bénéficiaire se trouve face à une quinzaine d'organisations internationales, des dizaines d'agences gouvernementales et des centaines d'ONG. Pour la seule Suisse, on trouve sur place des représentants du DFAE, de la DDC (et du Corps suisse d'aide en cas de catastrophe), de l'Office fédéral des réfugiés, du Département de la défense, de la protection de la population et des sports (DDPS) et d'une quinzaine d'ONG suisses. Un travail énorme reste à effectuer pour avoir une meilleure circulation des informations et améliorer la coordination entre ONG. 


\section{SOURCES}

Statistiques de l'aide humanitaire et actions de la Confédération et des ONG suisses

OCDE, Coopération pour le développement. Efforts et politiques des membres du Comité d'aide au développement, rapport 1996, OCDE, Paris, 1997.

$\mathrm{OCDE}$, Coopération pour le développement. Efforts et politiques des membres du Comité d'aide au développement, rapport 1997, OCDE, Paris, 1998.

«Message du Conseil fédéral concernant la continuation de l'aide humanitaire de la Confédération» du 20 novembre 1996, message 96.092 , Berne.

DDC, Rapport annuel Coopération internationale de la Suisse - Aide humanitaire 1997, DDC, Berne, 1998.

DDC, Corps suisse d'aide en cas de catastrophe (ASC), brochure de présentation, DDC, Berne, 1998, $64 \mathrm{p}$

IUED/DDC, Aide suisse aux pays en développement et aux pays de l'Europe orientale 1996/97, IUED, Genève, 1998 (contient la liste des ONG actives dans la coopération au développement et dans l'aide humanitaire, les données sur les montants d'aide versés par les ONG, ainsi que l'aide bilatérale de la Confédération par pays bénéficiaires).

Mosquito, Die entwicklungspolitische Zeitschrift der Schweiz, Nr. 4, Juni 1997 (Dossier: Spendenmarkt Schweiz: Umfang, Strategien, Gewinner, Verlierer).

IUED, Etude sur l'évolution de l'aide humanitaire des pays membres du CAD, IUED, Genève, 1993 (rapport pour le CICR, non publié)

Sur l'aide humanitaire en général

Claire Pirotte et Bernard Husson (dir.), Entre urgence et développement. Pratiques humanitaires en questions, Karthala, Paris, 1997, 238 p.

Myriam Donsimoni, Du don à l'aide. Le marché de l'altruisme, L'Harmattan, Paris, 1995, 228 p.

René Holenstein, Was kümmert uns die Dritte Welt. Zur Geschichte der internationalen Solidarität in der Schweiz, Chronos Verlag, Zürich, 1998, 264 p.

Myriam Tsikounas (dir.), Les ambiguïtés de l'humanitaire, Editions Corlet (diffusion Le Seuil), 1996, 190 p. (ouvrage collectif).

Ouvrage collectif, ONG et développement. Société, économie, politique, Karthala, Paris, 1998, 684 p.

"Coopération internationale: le temps des incertitudes ", dossier dans Revue Tiers Monde, $\mathrm{n}^{\circ} 151$, juillet-septembre 1997.

\section{SITES INTERNET}

DDC (Direction du développement et de la coopération): http//www.sdc-gov.ch

Corps suisse d'aide en cas de catastrophe: http//www.skh.ch 
Annexe I: Evolution de l'aide humanitaire de la Confédération (APD et AP), en millions de francs pour les principaux pays bénéficiaires

\begin{tabular}{|c|c|c|c|c|c|c|c|c|c|}
\hline Pays/région & 1990 & 1991 & 1992 & 1993 & 1994 & 1995 & 1996 & 1997 & Total \\
\hline \multicolumn{10}{|l|}{ Europe } \\
\hline Ex-Yougoslavie & 0.00 & 2.62 & 26.00 & 39.64 & 27.54 & 30.68 & 16.90 & 13.21 & 156.6 \\
\hline Russie & & & & 0.46 & 2.29 & 5.32 & 4.06 & 1.67 & 13.8 \\
\hline Albanie & & 0.73 & 2.08 & 0.59 & 0.22 & 0.54 & 0.04 & 0.44 & 4.6 \\
\hline \multicolumn{10}{|l|}{ Afrique } \\
\hline Rwanda & 0.34 & 0.96 & 5.55 & 10.19 & 20.18 & 16.92 & 13.37 & 7.51 & 75.0 \\
\hline Mozambique & 5.74 & 5.57 & 13.39 & 6.94 & 6.53 & 4.40 & 1.92 & 0.44 & 44.9 \\
\hline Angola & 5.62 & 2.43 & 1.92 & 3.96 & 4.88 & 4.18 & 9.41 & 8.30 & 40.7 \\
\hline Ethiopie & 4.07 & 12.78 & 3.53 & 3.92 & 2.17 & 5.31 & 5.58 & 2.30 & 39.7 \\
\hline Soudan & 5.77 & 4.84 & 4.88 & 3.56 & 2.33 & 4.32 & 3.25 & 3.03 & 32.0 \\
\hline Somalie & 2.45 & 4.98 & 5.06 & 2.74 & 0.79 & 2.34 & 1.55 & 3.84 & 23.7 \\
\hline Madagascar & 0.85 & 1.53 & 3.06 & 4.91 & 4.51 & 2.98 & 1.70 & 1.77 & 21.3 \\
\hline Congo (ex-Zaïre) & 0.63 & 2.29 & 0.60 & 1.17 & 3.22 & 1.17 & 4.77 & 2.29 & 16.1 \\
\hline Tanzanie & 1.36 & 0.86 & 1.88 & 0.93 & 5.62 & 0.68 & 1.05 & 2.12 & 14.5 \\
\hline Liberia & 0.20 & 0.50 & 2.04 & 0.28 & 2.53 & 3.53 & 2.40 & 1.62 & 13.1 \\
\hline Erythrée & & & & 0.00 & 4.23 & 4.39 & 1.61 & 1.56 & 11.8 \\
\hline Malawi & 2.85 & 3.63 & 2.58 & 0.27 & 1.46 & 0.40 & 0.24 & 0.11 & 11.5 \\
\hline Kenya & 0.05 & 1.78 & 2.57 & 1.72 & 1.69 & 1.46 & 0.78 & 0.97 & 11.0 \\
\hline Cap-Vert & 0.57 & 0.86 & 1.26 & 0.81 & 1.58 & 1.66 & 2.03 & 1.52 & 10.3 \\
\hline Algérie & 0.69 & 4.77 & 0.83 & 1.06 & 0.86 & 0.73 & 0.67 & 0.57 & 10.2 \\
\hline Afrique du Sud & 1.32 & 1.94 & 1.97 & 2.01 & 1.53 & 0.53 & 0.39 & 0.37 & 10.1 \\
\hline Ouganda & 2.30 & 2.34 & 2.84 & 0.27 & 0.20 & 0.13 & & & 8.1 \\
\hline Zimbabwe & 0.29 & 0.08 & 4.30 & 1.18 & 0.54 & 0.63 & 0.18 & 0.33 & 7.5 \\
\hline Burundi & 0.11 & 0.19 & 0.62 & 0.79 & 1.34 & 0.53 & 2.14 & 1.24 & 6.9 \\
\hline Burkina Faso & 0.50 & 1.46 & 1.30 & 0.79 & 0.56 & 0.68 & 1.03 & 0.58 & 6.9 \\
\hline Zambie & 0.49 & 0.83 & 1.73 & 0.62 & 0.58 & 1.03 & 0.39 & 0.38 & 6.1 \\
\hline Tchad & 0.26 & 1.98 & 0.25 & 0.84 & 0.71 & 0.30 & 0.18 & 0.30 & 4.8 \\
\hline Sénégal & 1.29 & 0.80 & 0.60 & 0.44 & 0.57 & 0.38 & 0.34 & 0.34 & 4.8 \\
\hline Mauritanie & 0.28 & 0.45 & 0.87 & 1.32 & 0.17 & 0.26 & 0.71 & 0.20 & 4.3 \\
\hline Mali & 0.05 & 0.85 & 0.26 & 0.02 & 0.13 & 0.10 & 0.94 & 1.59 & 3.9 \\
\hline Total Afrique & & & & & 71.80 & 61.69 & 58.68 & 46.81 & \\
\hline
\end{tabular}

\section{Asie/Moyen-Orient}

\begin{tabular}{llllllllll}
\hline Rép. du Caucase & & & & 1.02 & 5.70 & 5.02 & 4.44 & 8.20 & $\mathbf{2 4 . 4}$ \\
\hline Afghanistan & 2.70 & 3.61 & 5.41 & 2.00 & 2.00 & 3.80 & 1.80 & 2.70 & $\mathbf{2 4 . 0}$ \\
\hline Sri Lanka & 2.33 & 2.33 & 3.82 & 1.67 & 2.40 & 1.63 & 1.37 & 1.03 & $\mathbf{1 6 . 6}$ \\
\hline Israël & 2.01 & 3.83 & 2.18 & 3.30 & 1.61 & 0.50 & 0.03 & 0.50 & $\mathbf{1 4 . 0}$ \\
\hline Cambodge & 0.86 & 3.91 & 1.96 & 1.94 & 2.79 & 1.51 & 0.41 & 0.34 & $\mathbf{1 3 . 7}$ \\
\hline Corée du Nord & & & & 0.00 & 0.00 & 4.33 & 2.79 & 6.32 & $\mathbf{1 3 . 4}$ \\
\hline Liban & 2.40 & 2.56 & 1.36 & 1.17 & 1.40 & 0.72 & 1.54 & 1.10 & $\mathbf{1 2 . 3}$ \\
\hline Irak & & 1.79 & 2.34 & 0.06 & 0.11 & 1.11 & 2.81 & 2.54 & $\mathbf{1 0 . 8}$ \\
\hline Iran & 3.15 & 5.92 & 0.01 & 0.55 & 0.05 & & 0.04 & 0.52 & $\mathbf{1 0 . 2}$ \\
\hline Inde & 2.58 & 2.00 & 1.93 & 1.38 & 0.49 & 0.80 & 0.27 & 0.47 & $\mathbf{9 . 9}$ \\
\hline Jordanie & 0.68 & 8.37 & 0.00 & 0.00 & 0.00 & 0.50 & & & $\mathbf{9 . 5}$ \\
\hline Bangladesh & 0.93 & 2.57 & 3.14 & 0.53 & 0.76 & 0.58 & 0.41 & 0.27 & $\mathbf{9 . 2}$ \\
\hline Thaillande & 3.48 & 2.51 & 0.59 & 0.11 & 1.17 & 0.26 & 0.25 & 0.50 & $\mathbf{8 . 9}$ \\
\hline Palestine & & & & 3.00 & 0.69 & 1.76 & 1.39 & 0.78 & $\mathbf{7 . 6}$ \\
\hline Vietnam & 0.78 & 0.16 & 1.20 & 1.14 & 1.74 & 1.08 & 0.35 & 0.82 & $\mathbf{7 . 3}$ \\
\hline Tadjikistan & & & & 0.50 & 1.30 & 0.86 & 1.82 & 2.12 & $\mathbf{6 . 6}$ \\
\hline Philippines & 1.44 & 1.47 & 1.33 & 0.53 & 0.43 & 0.16 & 0.14 & 0.27 & $\mathbf{5 . 8}$ \\
\hline Népal & 0.53 & 0.23 & 0.39 & 0.86 & 1.37 & 0.70 & 0.22 & 0.15 & $\mathbf{4 . 4}$ \\
\hline
\end{tabular}




\begin{tabular}{lrrrrrrrrr}
\hline Pays/région & $\mathbf{1 9 9 0}$ & $\mathbf{1 9 9 1}$ & $\mathbf{1 9 9 2}$ & $\mathbf{1 9 9 3}$ & $\mathbf{1 9 9 4}$ & $\mathbf{1 9 9 5}$ & $\mathbf{1 9 9 6}$ & $\mathbf{1 9 9 7}$ & Total \\
\hline Indonésie & 0.26 & 0.00 & 0.03 & 0.32 & 0.61 & 0.35 & 0.19 & 0.68 & $\mathbf{2 . 4}$ \\
\hline Chine & 0.16 & 0.30 & 0.30 & 0.05 & 0.01 & 0.24 & 0.49 & 0.41 & $\mathbf{2 . 0}$ \\
\hline Total Asie & & & & & $\mathbf{2 7 . 2 9}$ & $\mathbf{2 6 . 2 6}$ & $\mathbf{2 1 . 6 3}$ & $\mathbf{2 9 . 9 2}$ & \\
\hline Amérique latine & & & & & & & & & \\
\hline Pérou & 4.47 & 5.08 & 4.73 & 3.27 & 3.03 & 2.12 & 2.24 & 1.99 & $\mathbf{2 6 . 9}$ \\
\hline Brésil & 2.05 & 1.73 & 2.33 & 2.09 & 2.03 & 2.07 & 1.37 & 1.34 & $\mathbf{1 5 . 0}$ \\
\hline Chili & 1.99 & 1.58 & 1.63 & 1.31 & 1.21 & 1.09 & 0.89 & 0.80 & $\mathbf{1 0 . 5}$ \\
\hline Haïti & 0.90 & 0.78 & 1.76 & 0.79 & 2.18 & 1.44 & 0.96 & 0.85 & $\mathbf{9 . 6}$ \\
\hline Colombie & 0.47 & 0.22 & 0.28 & 0.22 & 0.70 & 1.15 & 0.74 & 1.08 & $\mathbf{4 . 9}$ \\
\hline Guatemala & 0.23 & 0.53 & 1.19 & 0.07 & 0.63 & 0.54 & 0.51 & 0.64 & $\mathbf{4 . 3}$ \\
\hline Equateur & 0.21 & 0.36 & 0.43 & 0.49 & 0.82 & 0.75 & 0.72 & 0.47 & $\mathbf{4 . 2}$ \\
\hline Nicaragua & 0.95 & 0.44 & 0.54 & 0.61 & 0.80 & 0.21 & 0.15 & 0.14 & $\mathbf{3 . 8}$ \\
\hline Total Amérique latine & & & & $\mathbf{1 3 . 1 6}$ & $\mathbf{1 0 . 1 7}$ & $\mathbf{8 . 0 0}$ & $\mathbf{7 . 8 6}$ & \\
\hline Non ventilée géogr. & & & & $\mathbf{6 5 . 8 7}$ & $\mathbf{6 8 . 1 7}$ & $\mathbf{7 3 . 9 3}$ & $\mathbf{7 4 . 4 9}$ & \\
\hline Multilatérale & & & & & $\mathbf{4 4 . 6 8}$ & $\mathbf{4 1 . 2 2}$ & $\mathbf{4 6 . 4 6}$ & $\mathbf{4 4 . 6 0}$ & \\
\hline Total aide & & & & & $\mathbf{2 5 3 . 6 7}$ & $\mathbf{2 4 4 . 7 6}$ & $\mathbf{2 3 0 . 4 0}$ & $\mathbf{2 2 2 . 1 1}$ & \\
\hline Dont APD & 190.40 & 228.70 & 245.20 & 236.10 & 250.70 & 238.80 & 225.70 & 217.10 & \\
\hline Aide en \% APD & 19.06 & 20.37 & 18.11 & 19.72 & 19.27 & 19.32 & 17.83 & 17.84 & \\
\hline
\end{tabular}

Annexe II: Evolution de l'aide humanitaire des ONG, en millions de francs Principaux pays bénéficiaires des actions humanitaires des ONG

\begin{tabular}{lrrrrrrrrr}
\hline Pays/région & $\mathbf{1 9 9 0}$ & $\mathbf{1 9 9 1}$ & $\mathbf{1 9 9 2}$ & $\mathbf{1 9 9 3}$ & $\mathbf{1 9 9 4}$ & $\mathbf{1 9 9 5}$ & $\mathbf{1 9 9 6}$ & $\mathbf{1 9 9 7}$ & Total \\
\hline Europe & & & & & & & & & \\
\hline Ex-Yougoslavie & 0.00 & 1.22 & 4.41 & 11.84 & 22.87 & 16.35 & 18.47 & 13.42 & $\mathbf{8 8 . 6}$ \\
\hline Roumanie & 3.40 & 5.63 & 2.43 & 2.79 & 1.80 & 1.34 & 3.00 & 2.72 & $\mathbf{2 3 . 1}$ \\
\hline Turquie & 0.02 & 4.10 & 0.56 & 1.03 & 0.03 & 0.03 & 0.24 & 0.12 & $\mathbf{6 . 1}$ \\
\hline Russie & & 0.08 & 0.26 & 0.05 & 0.31 & 1.87 & 1.29 & 0.59 & $\mathbf{4 . 4}$ \\
\hline Bulgarie & 0.22 & 0.76 & 0.08 & 0.20 & 0.21 & 0.14 & 1.00 & 0.58 & $\mathbf{3 . 2}$ \\
\hline Pologne & 0.16 & 0.46 & 0.73 & 0.35 & 0.35 & 0.21 & 0.07 & 0.58 & $\mathbf{2 . 9}$ \\
\hline Albanie & & 0.12 & 0.22 & 0.26 & 0.25 & 0.22 & 0.54 & 1.11 & $\mathbf{2 . 7}$ \\
\hline Total Europe Sud $\mathbf{3 . 9 8}$ & $\mathbf{5 . 4 5}$ & $\mathbf{1 0 . 8 7}$ & $\mathbf{1 3 . 1 4}$ & $\mathbf{2 3 . 1 6}$ & $\mathbf{1 6 . 6 5}$ & $\mathbf{1 9 . 2 4}$ & $\mathbf{1 4 . 8 1}$ & \\
\hline Europe Est & & $\mathbf{1 2 . 4 9}$ & $\mathbf{7 . 3 9}$ & $\mathbf{4 . 0 1}$ & $\mathbf{3 . 0 6}$ & $\mathbf{3 . 8 7}$ & $\mathbf{6 . 0 0}$ & $\mathbf{5 . 9 9}$ & \\
\hline & & & & & & & & & \\
\hline Afrique & & & & & & & & & \\
\hline Rwanda & 0.10 & 0.07 & 0.44 & 0.57 & 10.19 & 5.03 & 2.77 & 2.94 & $\mathbf{2 2 . 1}$ \\
\hline Soudan & 0.39 & 1.64 & 1.23 & 1.60 & 2.73 & 1.28 & 2.21 & 3.10 & $\mathbf{1 4 . 2}$ \\
\hline Somalie & 0.00 & 0.03 & 3.55 & 3.95 & 1.71 & 1.55 & 1.64 & 1.76 & $\mathbf{1 4 . 2}$ \\
\hline Mozambique & 0.24 & 2.17 & 1.03 & 1.49 & 1.52 & 0.77 & 0.56 & 0.34 & $\mathbf{8 . 1}$ \\
\hline Ethiopie & 1.88 & 2.34 & 0.58 & 1.39 & 0.78 & 0.27 & 0.54 & 0.20 & $\mathbf{8 . 0}$ \\
\hline Madagascar & 0.58 & 0.48 & 0.92 & 0.63 & 0.70 & 0.64 & 1.10 & 1.09 & $\mathbf{6 . 1}$ \\
\hline Ouganda & 0.58 & 0.67 & 1.30 & 1.73 & 0.83 & 0.17 & 0.19 & 0.12 & $\mathbf{5 . 6}$ \\
\hline Angola & 0.60 & 0.56 & 0.70 & 0.52 & 0.44 & 1.00 & 0.93 & 0.69 & $\mathbf{5 . 4}$ \\
\hline Tanzanie & 1.13 & 0.40 & 0.58 & 0.65 & 1.29 & 0.44 & 0.31 & 0.40 & $\mathbf{5 . 2}$ \\
\hline Zimbabwe & 0.33 & 0.06 & 2.20 & 0.29 & 0.13 & 1.45 & 0.04 & 0.05 & $\mathbf{4 . 5}$ \\
\hline Burkina Faso & 0.64 & 1.71 & 0.02 & 0.05 & 0.06 & 0.00 & 0.18 & 0.04 & $\mathbf{2 . 7}$ \\
\hline Afrique du Sud & 0.21 & 0.35 & 0.25 & 0.45 & 0.23 & 0.30 & 0.15 & 0.54 & $\mathbf{2 . 5}$ \\
\hline Zambie & 0.33 & 0.16 & 0.36 & 0.22 & 0.59 & 0.25 & 0.37 & 0.17 & $\mathbf{2 . 5}$ \\
\hline Congo (ex-Zaire) & & 0.08 & 0.41 & 0.46 & 0.24 & 0.14 & 0.07 & 0.86 & $\mathbf{2 . 3}$ \\
\hline Kenya & 0.04 & 0.06 & 0.44 & 0.34 & 0.27 & 0.46 & 0.13 & 0.30 & $\mathbf{2 . 0}$ \\
\hline Burundi & 0.00 & 0.10 & 0.02 & 0.21 & 0.22 & 0.24 & 0.58 & 0.64 & $\mathbf{2 . 0}$ \\
\hline
\end{tabular}




\begin{tabular}{|c|c|c|c|c|c|c|c|c|c|}
\hline Pays/région & 1990 & 1991 & 1992 & 1993 & 1994 & 1995 & 1996 & 1997 & Tota \\
\hline Aide aux Sahraoui & is 0.06 & & 0.31 & 0.20 & 0.46 & 0.45 & 0.29 & 0.12 & 1.9 \\
\hline Erythrée & 0.74 & 0.00 & 0.11 & 0.22 & 0.18 & 0.11 & 0.01 & 0.14 & 1.5 \\
\hline Total Afrique & 9.57 & 13.39 & 16.58 & 18.04 & 23.79 & 16.31 & 14.85 & 15.96 & \\
\hline \multicolumn{10}{|c|}{ Asie/Moyen-Orient } \\
\hline Irak & 0.00 & 13.04 & 19.78 & 10.81 & 0.51 & 0.77 & 2.22 & 1.35 & 48.5 \\
\hline Inde & 1.87 & 1.57 & 2.11 & 1.94 & 1.22 & 1.43 & 3.21 & 2.61 & 15.9 \\
\hline Arménie & 0.16 & 5.24 & 3.54 & 1.06 & 0.68 & 1.52 & 0.45 & 0.36 & 13.0 \\
\hline Bangladesh & 0.37 & 3.98 & 0.65 & 2.12 & 0.34 & 0.31 & 2.09 & 2.62 & 12.5 \\
\hline Palestine/lsraël & 0.00 & 3.04 & 5.09 & 0.05 & 0.09 & 0.02 & 0.69 & 0.74 & 9.7 \\
\hline Philippines & 0.58 & 2.0 & 0.55 & 0.44 & 0.06 & 0.58 & 2.52 & 2.51 & 9.3 \\
\hline Liban & 0.96 & 1.1 & 1.15 & 0.89 & 0.12 & 0.20 & 1.21 & 1.17 & 6.8 \\
\hline Iran & 1.15 & 2.09 & 0.39 & 0.54 & 0.39 & & & 0.40 & 5.0 \\
\hline Total Asie & 5.88 & 28.31 & 31.84 & 20.88 & 5.26 & 7.58 & 16.39 & 15.10 & \\
\hline \multicolumn{10}{|l|}{ Amérique latine } \\
\hline Brésil & 0.34 & 0.91 & 0.55 & 0.39 & 0.45 & 0.48 & 1.52 & 0.55 & 5.2 \\
\hline Colombie & 0.34 & 0.19 & 0.44 & 0.27 & 0.27 & 0.73 & 0.41 & 0.61 & 3.2 \\
\hline Pérou & 0.26 & 0.25 & 0.51 & 0.11 & 0.15 & 0.19 & 0.78 & 0.85 & 3.1 \\
\hline Chili & 0.60 & 0.21 & 0.20 & 0.25 & 0.30 & 0.26 & 0.69 & 0.35 & 2.9 \\
\hline I Salvador & 1.16 & 0.41 & 0.58 & 0.35 & 0.23 & 0.03 & 0.02 & 0.00 & 2.8 \\
\hline Nicaragua & 0.73 & 0.32 & 0.40 & 0.47 & 0.20 & 0.17 & 0.14 & 0.05 & 2.5 \\
\hline Haïti & 0.08 & 0.08 & 0.12 & 0.24 & 0.17 & 0.42 & 0.49 & 0.43 & 2.0 \\
\hline Bolivie & 0.06 & 0.07 & 0.11 & 0.34 & 0.10 & 0.10 & 0.18 & 0.70 & 1.7 \\
\hline Guatemala & 0.49 & 0.02 & & 0.05 & 0.17 & 0.22 & 0.06 & 0.40 & 1.4 \\
\hline $\begin{array}{l}\text { Total } \\
\text { Amérique latine }\end{array}$ & 4.77 & 2.99 & 3.42 & 3.17 & 2.39 & 2.75 & 4.61 & 4.26 & \\
\hline Non ve & 3.28 & 0.14 & 0.10 & & 0.27 & 3.73 & 0.56 & 1.69 & \\
\hline Total aide Sud & 27.48 & 50.41 & 62.83 & 55.32 & 54.91 & 47.04 & 55.66 & 51.82 & \\
\hline Total aide Est & & 12.49 & 7.39 & 4.01 & 3.06 & 3.87 & 6.00 & 5.99 & \\
\hline $\begin{array}{l}\text { Contributions } \\
\text { DDC aux ONG }\end{array}$ & 30.57 & 28.05 & 42.44 & 39.90 & 40.40 & 38.10 & 30.50 & 32.26 & \\
\hline $\begin{array}{l}\text { Aide ONG Sud } \\
\text { (fonds propres) }\end{array}$ & 27.48 & 50.41 & 62.83 & 55.32 & 54.91 & 47.04 & 55.66 & 51.82 & \\
\hline Aide ONG Est & & 12.49 & 7.39 & 4.01 & 3.06 & 3.87 & 6.00 & 6.0 & \\
\hline Total ONG & & 90.95 & 112.65 & 99.23 & 98.37 & 89.01 & 92.16 & 90.08 & \\
\hline
\end{tabular}

Sources: DDC, Rapport annuel Coopération internationale de la Suisse - Aide humanitaire 1997, DDC, Berne, 1998 ainsi que les éditions précédentes de 1990 à 1997; IUED/DDC, Aide suisse aux pays en développement et aux pays de l'Europe orientale 1996/97, IUED, Genève, 1998 ainsi que les éditions précédentes de 1990 à 1997. 